\title{
On Approximate Equivalences of Multigranular Rough Sets and Approximate Reasoning
}

\author{
B. K. Tripathy \\ School of Computing Science and Engineering, VIT University, Vellore - 632014, Tamil Nadu, India \\ E-mail: tripathybk@vit.ac.in \\ Anirban Mitra \\ Department of Computer Science and Engineering, M.I.T.S., Kolonara, Rayagada - 765017, Odisha, India \\ E-mail: mitra.anirban@gmail.com
}

\begin{abstract}
The notion of rough sets introduced by Pawlak has been a successful model to capture impreciseness in data and has numerous applications. Since then it has been extended in several ways. The basic rough set introduced by Pawlak is a single granulation model from the granular computing point of view. Recently, this has been extended to two types of multigranular rough set models. Pawlak and Novotny introduced the notions of rough set equalities which is called approximate equalities. These notions of equalities use the user knowledge to decide the equality of sets and hence generate approximate reasoning. However, it was shown by Tripathy et al, even these notions have limited applicability to incorporate user knowledge. So the notion of rough equivalence was introduced by them. The notion of rough equalities in the multigranulation context was introduced and studied In this article, we introduce the concepts of multigranular rough equivalences and establish their properties. Also, the replacement properties, which are obtained by interchanging the bottom equivalences with the top equivalences, have been established. We provide a real life example for both types of multigranulation, compare the rough multigranular equalities with the rough multigranular equivalences and illustrate the interpretation of the rough equivalences through the example.
\end{abstract}

Index Terms - Rough Sets, Multigranular Rough Sets, Approximate Equivalences, Approximate Reasoning

\section{Introduction}

Impreciseness has become a common feature in modern day databases. Rough set [4, 5, 6] have been found to be a fruitful model for such type of data and also rough set techniques have been effective in the study of such databases in the form of rule generation, anonymisation and reduction of their size. Since then the basic notion of rough set has been extended in several ways $[7,8,9,10,12,16]$.
The basic notion of equality of two sets is independent of the user or more precisely the user knowledge about the universe dealt with. In an attempt to incorporate the user knowledge about the structure of the universe in concluding about the equality of two sets the notions of approximate equalities were introduced by Novotny and Pawlak ([1, 2, 3]). This is an important feature as the sets considered may not be equal in the normal sense but they have close features to assume that they are approximately equal. That is, basing upon our knowledge and requirement we can assume that the two sets are indistinguishable. Properties of approximate equalities established by Novotny and Pawlak $[1,2,3]$ were analysed in $[13,14]$ and it was shown that some of the observations there are not true. In [14] attempts were made to extend these properties to the generalised situation of rough equivalences. It was found that the properties failed to hold in their full generalities and mostly parts were found to hold true.

The other parts were established under suitable conditions. The validity of some basic algebraic properties involving union, intersection and complementation of sets were tested for their validity with equality of sets being replaced with rough equivalence in [11, 14]. Two types of Multig ranulations were introduced as extensions of single granulation. In fact optimistic multigranulation was introduced in [8] and pessimistic multigranulation was introduced in [9]. In this paper we confine ourselves to the extension of the approximate equivalences of Tripathy et al [11, 14] to the setting of multig ranulations and establish their direct properties and replacement properties also.

This paper consists of seven sections. First section presents the overview and related literatures. In section two, we have discussed on basic definitions and properties of rough sets and multigranular rough set followed by a real life example. The third section emphasis on some of the previous results in this direction with their comparison. Approximate Equalities and its properties are discussed in forth section. Fifth and sixth section discuss about Multigranular Rough equalities and Multigranular 
Rough equivalence, followed by conclusion in the seventh section. Lastly, we have presented the list of referred paperunder reference section.

\section{Definitions and Properties}

Let $\mathrm{U}$ be a universe of discourse and $\mathrm{R}$ be an equivalence relation over $U$. By $U / R$ we denote the family of all equivalence class of $\mathrm{R}$, referred to as categories or concepts of $\mathrm{R}$ and the equivalence class of an element $\mathrm{x} \in \mathrm{U}$, is denoted by $[\mathrm{x}]_{\mathrm{R}}$. By a knowledge base, we understand a relational system $\mathrm{K}=(\mathrm{U}, \mathrm{R})$, where $\mathrm{U}$ is as above and $\mathrm{R}$ is a family of equivalence relations over $\mathrm{U}$. For any subset $\mathrm{P}(\neq$ Let $U$ be a universe of discourse and $R$ be an equivalence relation over $U$. By $U / R$ we denote the family of all equivalence class of $R$, referred to as categories or concepts of $R$ and the equivalence class of an element $\mathrm{x} \in \mathrm{U}$, is denoted by $[\mathrm{x}]_{R}$. By a knowledge base, we understand a relational system $K=(U, R)$, where $U$ is as above and $R$ is a family of equivalence relations over $U$. For any subset $P(\neq \phi) \subseteq R$, the intersection of all equivalence relations in $P$ is denoted by IND (P) and is called the indiscernibility relation over $P$.

Given any $X \subseteq U$ and $R \in \operatorname{IND}(\mathrm{K})$, we as sociate two subsets,

$$
\underline{R X}=\bigcup\{\mathrm{Y} \in \mathrm{U} / \mathrm{R}: \mathrm{Y} \subseteq \mathrm{X}\}
$$

and

$$
\bar{R} X=\bigcup\{\mathrm{Y} \in \mathrm{U} / \mathrm{R}: \mathrm{Y} \cap \mathrm{X} \neq \phi\},
$$

called the R-lower and R-upper approximations of X respectively.

The R-boundary of $X$ is denoted by $\mathrm{BN}_{\mathrm{R}}(\mathrm{X})$ and is given by $\mathrm{BN}_{\mathrm{R}}(\mathrm{X})=\overline{\mathrm{R}} \mathrm{X}-\underline{\mathrm{RX}}$. The elements of $\underline{R} X$ are those elements of $U$, which can certain ly be classified as elements of $\mathrm{X}$, and the elements of $\bar{R} X$ are those elements of $U$, which can possibly be classified as elements of $\mathrm{X}$, employing knowledge of $R$. We say that $X$ is rough with respect to $R$ if and only if $\underline{R} X \neq \bar{R} X$, equivalently $\mathrm{BN}_{\mathrm{R}}(\mathrm{X}) \neq \phi . X$ is said to be R-definable if and only if $\underline{R} X=\bar{R} X$, or $\mathrm{BN}_{\mathrm{R}}(\mathrm{X})=\phi$.

\subsection{Multigranular Rough sets}

The concept of granular computing was introduced by Zadeh. According to this concept an equivalence relation on the universe can be regarded as a granulation, and a partition on the universe can be regarded as a granulation space. As mentioned earlier, from the granular computing point of view, two types of Multigranulations have been defined using rough sets.
The optimistic multigranular rough sets were introduced by Qian as follows. We note that in the beginning there was only one type of Multigranulation and it was not named as optimistic. After the development of a second type of Multigranulation, the first one was called optimistic and the second one was called as pessimistic. We note that we are considering double granulation only. For granulations of higher order, the definitions and properties are similar. The notations used for the two types of Multigranulations were different in the original papers. But we follow the notations used in a recent paper by Tripathy et al [17], that is, we use $\mathrm{R}+\mathrm{S}$ for optimistic Multigranulation and $R * S$ for pessimistic Multigranulation, where $\mathrm{R}$ and $\mathrm{S}$ are two equivalence relations on $\mathrm{U}$.

Definition 2.1.1: Let $K=(U, \mathbf{R})$ be a knowledge base, $\mathbf{R}$ be a family of equivalence relations, $\mathrm{X} \subseteq \mathrm{U}$ and $R, S \in \mathbf{R}$. We define the optimistic multi-granular lower approximation and optimistic multi-granular upper approximation of $\mathrm{X}$ with respect to $\mathrm{R}$ and $\mathrm{S}$ in $\mathrm{U}$ as

$$
\begin{aligned}
& \text { (2.1.1) } \underline{R+S} X=\left\{x \mid[x]_{R} \subseteq X \text { or }[x]_{S} \subseteq X\right\} \\
& \text { (2.1.2) } \overline{R+S} X=\sim(\underline{R+S}(\sim X))
\end{aligned}
$$

Definition 2.1.2: Let $K=(U, \mathbf{R})$ be a knowledge base, $\mathbf{R}$ be a family of equivalence relations, $\mathrm{X} \subseteq \mathrm{U}$ and $R, S \in \mathbf{R}$. We define the pessimistic multi-granular lower approximation and pessimistic multi-granular upper approximation of $\mathrm{X}$ with respect to $\mathrm{R}$ and $\mathrm{S}$ in $\mathrm{U}$ as

$$
\begin{aligned}
& \text { (2.1.3) } \underline{R^{*} S} X=\left\{x \mid[x]_{R} \subseteq X \text { and }[x]_{S} \subseteq X\right\} \\
& \text { (2.1.4) } \overline{R^{*} S} X=\sim\left(\underline{R^{*} S}(\sim X)\right) .
\end{aligned}
$$

\subsubsection{A real life Example for Multigranulations}

Let us consider the example of cattle $\mathrm{C}$ in a locality. We define a relation $\mathrm{R}$ over $\mathrm{C}$ set by $\mathrm{x} \mathrm{R} \mathrm{y}$ if and only if $\mathrm{x}$ and $\mathrm{y}$ are cattle of the same kind. Suppose, for example this equivalence relation decomposes the universe of cattle into disjoint equivalence classes given by $\mathrm{C}=\{$ Cow, Buffalo, Goat, Sheep, Bullock $\}$. Next we define another equivalence relation $S$ as $x \mathrm{~S} y$ if and only if $\mathrm{x}$ and $\mathrm{y}$ are of the same size. We get three equivalence classes as $\mathrm{U}=\{$ Small, Middle, Large $\}$. These are defined as

$$
\begin{aligned}
& \text { Large }=\{\text { Buffalo, Bullock }\}, \\
& \text { Middle }=\{\text { Cow }\} \text { and } \\
& \text { Small }=\{\text { Goat }, \text { Sheep }\} .
\end{aligned}
$$

Then for any subset $\mathrm{X}$ of the cattle in the society, we have: 
$\underline{R+S} X=\mathrm{It}$ is the set of cattle whose category is completely in $\mathrm{X}$ or all the cattle of its size are contained in X.

$\underline{R * S X}=$ It is the set of cattle whore category is completely in $X$ and all the cattle of its size are contained in $\mathrm{X}$.

$\overline{R+S} X=\mathrm{It}$ is the set of cattle whose category has nonempty intersection with $\mathrm{X}$ or whose size has nonempty intersection with $\mathrm{X}$.

$\overline{R * S} X=$ It is the set of cattle whose category has nonempty intersection with $\mathrm{X}$ and whose size has nonempty intersection with $\mathrm{X}$.

\subsection{Properties of Multigranulations}

We present below some properties of multigranulations which shall be used in this paper to establish the results.

\subsubsection{Properties of Optimistic Multigranular Rough Sets}

The following properties of the optimistic multigranular rough sets were established in [8].

(2.2.1.1) $\quad \underline{(\mathrm{R}+\mathrm{S})}(\mathrm{X}) \subseteq \mathrm{X} \subseteq \overline{(\mathrm{R}+\mathrm{S})}(\mathrm{X})$

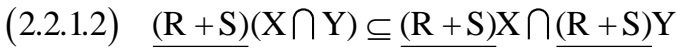

(2.2.1.3) $\overline{\overline{(R+S)}}(X \cup Y) \supseteq \overline{(R+S)} X \cup \overline{(R+S)} Y$

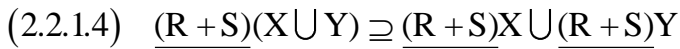

(2.2.1.5) $\overline{(\mathrm{R}+\mathrm{S})}(\mathrm{X} \cap \mathrm{Y}) \subseteq \overline{(\mathrm{R}+\mathrm{S})} \mathrm{X} \cap \overline{(\mathrm{R}+\mathrm{S}) \mathrm{Y}}$

(2.2.1.6) $\quad \underline{(\mathrm{R}+\mathrm{S})}(\mathrm{X})=\underline{\mathrm{R} X} \cap \underline{\mathrm{S} X}$

(2.2.1.7) $\overline{(R+S)}(X)=\bar{R} X \bigcup \bar{S} X$

\subsubsection{Properties of Pessimistic Multigranular Rough sets}

The following properties of the pessimistic multigranular rough sets which are parallel to the properties in 3.1 were established in [9].

$$
\underline{(\mathrm{R} * \mathrm{~S})}(\mathrm{X}) \subseteq \mathrm{X} \subseteq \overline{(\mathrm{R} * \mathrm{~S})}(\mathrm{X})
$$

(2.2.2.2) $\quad(\mathrm{R} * \mathrm{~S})(\mathrm{X})=\underline{\mathrm{R} X \cap S \mathrm{X}}$

(2.2.2.3) $\left(\overline{R^{*} S}\right)(X)=\bar{R} X \bigcup \bar{S} X$

(2.2.2.4) $\quad \underline{(\mathrm{R} * \mathrm{~S})}(\mathrm{X} \cap \mathrm{Y}) \subseteq(\mathrm{R} * \mathrm{~S}) \mathrm{X} \cap(\mathrm{R} * \mathrm{~S}) \mathrm{Y}$

(2.2.2.5) $(\overline{\mathrm{R} * \mathrm{~S}})(\mathrm{X} \cup \mathrm{Y}) \supseteq(\overline{\mathrm{R} * \mathrm{~S}}) \mathrm{X} \cup(\overline{\mathrm{R} * \mathrm{~S}}) \mathrm{Y}$

(2.2.2.6) $(\underline{\mathrm{R} * \mathrm{~S}})(\mathrm{X} \cup \mathrm{Y}) \supseteq(\underline{\mathrm{R} * \mathrm{~S}}) \mathrm{X} \cup(\underline{\mathrm{R} * \mathrm{~S}}) \mathrm{Y}$

(2.2.2.7) $\overline{(\mathrm{R} * \mathrm{~S})}(\mathrm{X} \cap \mathrm{Y}) \subseteq \overline{(\mathrm{R} * \mathrm{~S})} \mathrm{X} \cap \overline{(\mathrm{R} * \mathrm{~S})} \mathrm{Y}$

\section{Some Previous Results}

In this section, we provide some results on multigranular rough sets, which are to be used in our work.

\subsection{Comparison Results}

Among the properties (2.2.1.4) to (2.2.1.7) and (2.2.2.4) to (2.2.2.7), in the single granulation case (2.2.1.4), (2.2.1.5), (2.2.2.4) and (2.2.2.5) are actually equalities. However, it has been established in $[17,19]$ that inclusions in (2.2.2.4) and (2.2.2.5) can be replaced with equalities. That is

$$
\begin{aligned}
& \text { (3.1.1) } \quad \underline{(\mathrm{R} * \mathrm{~S})}(\mathrm{X} \cap \mathrm{Y})=\underline{(\mathrm{R} * \mathrm{~S}) \mathrm{X} \cap(\mathrm{R} * \mathrm{~S}) \mathrm{Y}} \\
& \text { (3.1.2) } \quad\left(\overline{R^{*} S}\right)(X \cup Y)=\left(\overline{R^{*} S}\right) X \cup\left(\overline{R^{*} S}\right) Y
\end{aligned}
$$

However, it has also been shown in $[17,19]$ that the inclusions in (2.2.1.4) to (2.2.1.7), (2.2.2.6) and (2.2.2.7) cannot be replaced with equalities. So, we can say that the deviations are in the properties (2.2.1.4) and (2.2.1.5) from the base case.

\subsection{Algebraic properties of Multigranulations}

In this section we establish some algebraic properties of both types of Multigranulations. It is interesting to find conditions or the cases under which the two types of Multigranulations reduce to single granulation rough sets. In this section we present two results in this direction established in [19].

Theorem 3.2.1: Let $\mathrm{R}$ and $\mathrm{S}$ be two equivalence relations on $\mathrm{U}$ and $X \subseteq U$. Then

(3.2.1) $\underline{\mathrm{R}+\mathrm{S}} \mathrm{X}=\underline{\mathrm{R}} \mathrm{X}$ and $\overline{\mathrm{R}+\mathrm{S}} \mathrm{X}=\overline{\mathrm{R}} \mathrm{X}$ when $\mathrm{S}=U \times U$.

(3.2.2) $\underline{\mathrm{R} * \mathrm{~S} X}=\underline{\mathrm{R} X}$ and $\overline{\mathrm{R} * \mathrm{~S} X}=\overline{\mathrm{R}} \mathrm{X}$ when $S=\{(x, x) \mid x \in U\}$.

Theorem 3.2.2: With the same notations as in Theorem 3.3.1, the following properties [18] are satisfied by ' + ' and '*':

(3.2.3) $\begin{aligned} & \frac{R+S}{\overline{R+S}} X=\overline{S+R} X \text { and } \\ & \overline{S+R} X\end{aligned}$

(3.2.4) $\underline{(R+S)+T X}=\underline{R+(S+T) X}$ and

$\overline{(R+S)+T} X=\overline{R+(S+T)} X$ 
(3.2.6)

$$
\begin{aligned}
& \frac{(R * S) * T X}{\overline{(R * S) * T}} X=\underline{R *(S * T)} X \text { and } \\
& \overline{R *(S * T)} X
\end{aligned}
$$

Let us denote the pessimistic Multig ranular rough sets and the optimistic rough sets associated with $\mathrm{R}$ and $S$ by and $R+S$ respectively. $R * S$

Theorem 3.2.3: For any and two equivalence relations $\mathrm{R}$ and $\mathrm{S}$ defined over $\mathrm{U}$ and any $X \subseteq U$ we have

$$
\begin{aligned}
& \frac{R * S}{\subseteq} \subseteq \underline{R+S} X \subseteq \underline{R \cap S} X \subseteq \overline{R \cap S} X \\
& \subseteq \overline{R+S} X \subseteq \overline{R * S} X
\end{aligned}
$$

From the above theorem the following result has been derived in [17]. This result provides a comparison among the three types of multigranulations including the intersection of the two equivalence relations which is also an equivalence relation.

Corollary 3.2.1: For any and two equivalence relations $\mathrm{R}$ and $\mathrm{S}$ defined over $\mathrm{U}$ and any $X \subseteq U$ we have

$$
\begin{aligned}
& B N(R \cap S)(X) \subseteq B N(R+S)(X) \\
& \subseteq B N(R * S)(X)
\end{aligned}
$$

(3.2.9) $\mathrm{X}$ is rough with respect to $R \cap S \Rightarrow \mathrm{X}$ is rough with respect to $R+S \Rightarrow \mathrm{X}$ is rough with respect to $R * S$.

(3.2.10) $\mathrm{X}$ is crisp with respect to $R * S \Rightarrow \mathrm{X}$ is crisp with respect to $R+S \Rightarrow \mathrm{X}$ is crisp with respect to $R \cap S$.

Theorem 3.2.4: A result which is not true for single granulation is also not true for multigranulation of any kind.

Proof: Suppose some result is not true for lower approximation or upper approximation with respect to single granulation R say. If the same result is true for mu ltigranulation (optimistic or pessimistic) with respect to two granulations $\mathrm{R}$ and $\mathrm{S}$ then by taking $\mathrm{S}=U \times U$ or $\mathrm{S}=\{(\mathrm{x}, \mathrm{x}) / x \in U\}$, it reduces to lower or upper approximations with respect to $\mathrm{R}$ only by Theorem 3.2.1. But by our assumption the result is not true in this case. So, our claim is true.

Theorem 3.2.5: $[\bar{R} X \cap \bar{S} Y]=\phi$ and $[\bar{R} Y \cap \bar{S} X]=\phi$ is a sufficient but not necessary condition for $\overline{R+S}(X \cup Y)=(\overline{R+S} X) \cup(\overline{R+S} Y)$.
Theorem 3.2.6: $\underline{\mathrm{RX}} \cup \underline{S Y}=\mathrm{U}$ and $\underline{\mathrm{R} Y} \cup \underline{\mathrm{S} X}=\mathrm{U} \quad$ is $\quad$ a sufficient but not necessary condition for

$$
\underline{\mathrm{R}+\mathrm{S}}(\mathrm{X} \cap \mathrm{Y})=\underline{\mathrm{R}+\mathrm{SX}} \cap \underline{\mathrm{R}+\mathrm{SY}} .
$$

\section{Approximate Equalities}

As described in the introduction, sometimes exact equality (equality in the mathematical sense) is too stringent to apply in day to day life. We often talk about equality of sets or domains, which can be considered to be equal for the purpose or under the circumstances in real life situations. So, approximate equalities play a significant role in our reasoning. Also, it is dependent upon the knowledge the assessors have about the domain under consideration as a whole but mostly not the knowledge about individuals.

\subsection{Rough Equalities}

As a step to incorporate user knowledge in considering equality of sets, Novotny and Pawlak [1, 2, 3] introduced the following.

Let $\mathrm{K}=(\mathrm{U}, \mathbf{R})$ be a knowledge base, $X, Y \subseteq U$ and $R \in I N D(K)$.

Definition 4.1.1: We say that:

(4.1.1) $\mathrm{X}$ and $\mathrm{Y}$ are bottom rough equal (X b_R_eq $\mathrm{Y}$ ) if and only if $\underline{R} X=\underline{R} Y$.

(4.1.2) $\mathrm{X}$ and $\mathrm{Y}$ are top rough equal (X t_R_eq $\mathrm{Y}$ ) if and only if $\bar{R} X=\bar{R} Y$.

(4.1.3) $\mathrm{X}$ and $\mathrm{Y}$ are rough equal (X R_eq $\mathrm{Y}$ ) if and only if (X b_R_eq $Y)$ and $\left(X t \_R \_e q Y\right)$.

Also 11 properties of these rough equalities were established and it was mentioned that these properties do not hold when bottom rough equality and top rough equality are interchanged. The later properties are called replacement properties. However, it is shown by Tripathy et al $[11,14]$ that some of these properties hold under the interchange where as some others hold with some additional conditions which are sufficient but not necessary.

As noted by Pawlak ([5], p.26), all these approximate equalities of sets are of relative character, that is things are equal or not equal from our point of view depending on what we know about them. So, in a sense the definition of rough equality refers to our knowledge about the universe. 


\section{Multigranular Rough Equalities}

In this section we shall define multig ranular rough equalities and establish both the direct as well as the replacement properties for these notions. Also, we shall extend the example of cattle to illustrate the concepts. In fact we shall introduce two types of multigranular rough equalities; namely the optimistic multigranular rough equalities and the pessimistic multig ranular rough equalities. In the next subsection we shall consider the pessimistic one and in the next section we shall take the other concept.

The notions of pessimistic multigranular rough equalities were introduced and studied in [20]. We present below the definitions.

Definition 5.1.1: Let $\mathrm{R}$ and $\mathrm{S}$ be two equivalence relations on $\mathrm{U}$ and $X, Y \subseteq U$. Then

(5.1.1) $\mathrm{X}$ and $\mathrm{Y}$ are pessimistic bottom rough equal to each other with respect to $\mathrm{R}$ and $\mathrm{S}\left(\mathrm{X} \mathrm{Pb} \mathrm{R}^{*} \mathrm{~S} \_\right.$eq $\left.\mathrm{Y}\right)$ if and only if $\underline{R * S} X=\underline{R * S Y}$.

(5.1.2) $\mathrm{X}$ and $\mathrm{Y}$ are pessimistic top rough equal to each other with respect to $\mathrm{R}$ and $\mathrm{S}$ (X Pt_R*S_eq Y) if and only if $\overline{R * S} X=\overline{R * S} Y$.

(5.1.3) $\mathrm{X}$ and $\mathrm{Y}$ are pessimistic rough equal to each other with respect to $\mathrm{R}$ and $\mathrm{S}$ ( $\mathrm{X} P \_\mathrm{R} * \mathrm{~S} \mathrm{Y}$ ) if and only if $\underline{R * S} X=\underline{R * S} Y$ and $\overline{R * S} X=\overline{R * S} Y$.

The notions of different optimistic multigranular rough equalities were introduced and studied in [20]. We present below the definitions.

Definition 5.2.1: Let $\mathrm{R}$ and $\mathrm{S}$ be two equivalence relations on $\mathrm{U}$ and $X, Y \subseteq U$. Then

(5.2.1) $\mathrm{X}$ and $\mathrm{Y}$ are optimistic bottom rough equal to each other with respect to $\mathrm{R}$ and $\mathrm{S}$ (X Ob_R+S_eq $\mathrm{Y}$ ) if and only if $\underline{R+S} X=\underline{R+S Y}$.

(5.2.2) $\mathrm{X}$ and $\mathrm{Y}$ are optimistic top rough equal to each other with respect to $\mathrm{R}$ and $\mathrm{S}$ (X Ot_R+S_eq $\mathrm{Y}$ ) if and only if $\overline{R+S} X=\overline{R+S} Y$.

(5.2.3) $\mathrm{X}$ and $\mathrm{Y}$ are optimistic rough equal to each other with respect to $\mathrm{R}$ and $\mathrm{S}$ ( $\mathrm{X}$ O_R+S Y) if and only if $\underline{R+S} X=\underline{R+S} Y$ and $\overline{R+S} X=\overline{R+S} Y$.

\section{Multigranular Rough Equivalences}

In this section we shall define multigranular rough equivalences and establish both the direct as well as the replacement properties for these notions. Also, we shall use the extended example of cattle in [20] to illustrate the concepts. In fact we shall introduce two types of multigranular rough equivalences; namely the optimistic multigranular rough equivalences and the pessimistic multigranular rough equivalences. In the next subsection we shall consider the pessimistic one and in the next section we shall take the other concept.

\subsection{Pessimistic Multigranular Rough Equivalences}

Definition 6.1.1: Let $\mathrm{R}$ and $\mathrm{S}$ be two equivalence relations on $\mathrm{U}$ and $X, Y \subseteq U$. Then,

(6.1.1) $\mathrm{X}$ and $\mathrm{Y}$ are pessimistic bottom rough equivalent to each other with respect to $\mathrm{R}$ and $\mathrm{S}$ ( $\mathrm{X}$ $\mathrm{Pb} \_\mathrm{R} * \mathrm{~S} \_$eqv $\left.\mathrm{Y}\right)$ if and only if $\underline{R * S} X$ and $\underline{R * S Y} Y$ are $\phi$ or not $\phi$ together.

(6.1.2) $\mathrm{X}$ and $\mathrm{Y}$ are pessimistic top rough equivalent to each other with respect to $\mathrm{R}$ and $\mathrm{S}$ (X Pt_R*S_eqv $\mathrm{Y}$ ) if and only if $\overline{R * S} X$ and $\overline{R * S} Y$ are equal to $\mathrm{U}$ or not $\mathrm{U}$ together.

(6.1.3) $\mathrm{X}$ and $\mathrm{Y}$ are pessimistic rough equivalent to each other with respect to $\mathrm{R}$ and $\mathrm{S}$ ( $\mathrm{X} \mathrm{P}_{-} \mathrm{R} * \mathrm{~S}$ _eqv $\mathrm{Y}$ ) if and only if $R * S X$ and $R * S Y$ are $\phi$ or not $\phi$ together and $\overline{R * S} X$ and $\overline{R * S} Y$ are equal to $\mathrm{U}$ or not $\mathrm{U}$ together.

The following properties hold for pessimistic Multigranular Rough Equivalences. In order to establish these properties we need the following additional definitions.

Definition 6.1.2: Let $\mathrm{K}=(\mathrm{U}, \mathfrak{R})$ be a knowledge base, $\mathrm{X}, \mathrm{Y} \subseteq \mathrm{U}$ and $\mathrm{R}, \mathrm{S} \in \mathrm{IND}(\mathrm{K})$. Then

(i) We say that $\mathrm{X}$ is bottom $(R * S)$-included in $\mathrm{Y}$ $\left(\mathrm{X} \sim_{R * S} \mathrm{Y}\right)$ if and only if $\underline{\mathrm{R} * \mathrm{SX}} \subseteq \underline{\mathrm{R} * \mathrm{SY}}$

(ii) $\mathrm{We}$ say that $\mathrm{X}$ is $\operatorname{top}(R * S)$-included in $\mathrm{Y}$ $\left(X \tilde{\subset}_{R * S} Y\right)$ if and only if $\overline{R * S} \mathrm{X} \subseteq \overline{R * S} \mathrm{Y}$.

(iii) $\mathrm{We}$ say that $\mathrm{X}$ is $\left(R^{*} S\right)$-included in $\mathrm{Y}\left(X{\underset{\sim}{\sim}}_{R * S} Y\right)$ if and only if $\mathrm{X} \sim_{R * S} \mathrm{Y}$ and $X \tilde{\subset}_{R * S} Y$

Definition 6.1.3: Let $\mathrm{K}=(\mathrm{U}, \mathfrak{R})$ be a knowledge base, $\mathrm{X}, \mathrm{Y} \subseteq \mathrm{U}$ and $\mathrm{R}, \mathrm{S} \in \mathrm{IND}(\mathrm{K})$. Then

(i) we say $\mathrm{X}, \mathrm{Y} \subseteq \mathrm{U}$ are bottom $R * S$ comparable if and only if $\mathrm{X}$ is bottom $\left(R^{*} S\right)$-included in $\mathrm{Y}$ or $\mathrm{Y}$ is $\operatorname{bottom}(R * S)$-included in X. 
(ii) We say $\mathrm{X}, \mathrm{Y} \subseteq \mathrm{U}$ are $\operatorname{top}(R * S)$ comparable if and only if $\mathrm{X}$ is $\operatorname{top}\left(R^{*} S\right)$-included in $\mathrm{Y}$ or $\mathrm{Y}$ is top $(R * S)$-included in $\mathrm{X}$.

(iii) We say $\mathrm{X}, \mathrm{Y} \subseteq \mathrm{U}$ are $(\mathrm{R} * \mathrm{~S})$ comparable if and only if $\mathrm{X}$ and $\mathrm{Y}$ are both top and bottom $\left(\mathrm{R}^{*} \mathrm{~S}\right)$ comparable.

Example: Let us consider the example of cattle above. Let P1 and P2 be two people in the locality having cattle set owned by them as $\mathrm{X}$ and $\mathrm{Y}$ respectively. There are four different cases in which we can talk about equivalence of $\mathrm{P} 1$ and $\mathrm{P} 2$.

Case I: $\overline{R * S} X, \overline{R * S} Y$ are not $\mathrm{U}$ and $\underline{R * S} X, \underline{R^{*} S Y}$ are $\phi$. Both $\mathrm{P} 1$ and $\mathrm{P} 2$ have some kind of cattle of some size but do not have all cattle of any kind or any size. So, they are equivalent.

Case II: $\overline{R^{*} S} X, \overline{R^{*} S} Y$ are not $\mathrm{U}$ and $\underline{R} \boldsymbol{*} X, \underline{R} * S Y$ are not $\phi$. Both $\mathrm{P} 1$ and $\mathrm{P} 2$ have some kind of cattle of some size and have all cattle of some kind or some size. So, they are equivalent.

Case III: $\overline{R^{*} S} X, \overline{R^{*} S} Y$ are $\mathrm{U}$ and $\underline{R * S} X, \underline{R^{*} S Y}$ are $\phi$. Both $\mathrm{P} 1$ and $\mathrm{P} 2$ have some cattle of every kind and some cattle of every size but do not have all cattle of any kind or any size. So, they are equivalent.

Case IV: $\overline{R * S} X, \overline{R * S} Y$ are $\mathrm{U}$ and $\underline{R * S} X, \underline{R * S Y}$ are not $\phi$. Both $\mathrm{P} 1$ and $\mathrm{P} 2$ have some cattle of every kind and some cattle of every size and also have all cattle of some kind and of some size. So, they are equivalent.

The following cases $\mathrm{P} 1$ and $\mathrm{P} 2$ are not considered to be equivalent:

Case V: One of $\overline{R^{*} S} X, \overline{R^{*} S} Y$ is $\mathrm{U}$ and the other one is not. Then out of P1 and P2 one has some cattle of every kind and some cattle of every size where as the other one does not have any cattle of some kind or some size. So, they are not equivalent.

Case VI: One of $\underline{R^{*} S} X, \underline{R^{*} S Y}$ is $\phi$ and the other one is not. Then one of $\mathrm{P} 1$ and $\mathrm{P} 2$ has all cattle of some kind and all cattle of some size where as the other one neither has all cattle of any kind nor all cattle of any size. So, they are not equivalent.

\section{Properties 6.1:}

(6.1.4) (i) If $X \cap Y$ is $\mathrm{b} \_\mathrm{R} * \mathrm{~S} \_$eqv to $\mathrm{X}$ and $X \cap Y$ is b_R*S_eqv to $Y$ then $X$ is $b \_R * S \_e q v$ to $Y$.

(ii) The converse of (i) is not necessarily true. (iii) The converse is true if in addition $\mathrm{X}$ and $\mathrm{Y}$ are bottom $\mathrm{R} * \mathrm{~S}$ comparable.

(iv) The condition in (iii) is not necessary.

Proof: Proof of (i) is trivial. Since the converse is not true in the base case, by Theorem 3.2.4 it is not true in this case. Proof of (iii) follows from (3.1.1).

Proof of (iv): We consider the same example as in [19] and take $\mathrm{S}=\{(\mathrm{x}, \mathrm{x}) / x \in U\}$.

Here, $[x]_{S}=\{x\}, \forall x \in U$.

So that $\underline{R * S} X=X \neq \phi, \underline{R * S Y} Y=Y \neq \phi$.

But $\underline{R * S}(X \cap Y)=\phi$.

(6.1.5) (i) If $X \cup Y$ is t_R* $\mathrm{S} \_$eqv to $\mathrm{X}$ and $X \cup Y$ is t_R*S_eqv to $Y$ then $X$ is $t \_R * S \_e q v$ to $Y$.

(ii) The converse of (i) is not necessarily true.

(iii) The converse is true if in addition $\mathrm{X}$ and $\mathrm{Y}$ are top $\mathrm{R}^{*} \mathrm{~S}$ comparable.

(iv) The condition in (iii) is not necessary.

Proof: Proof of (i) is trivial. Since the converse is not true in the base case, by Theorem 3.2.4 it is not true in this case. Proof of (iii) follows from (3.1.2).

Proof of (iv) follows as in (6.1.4) (iv).

(6.1.6) (i) If $\mathrm{X}$ is $\mathrm{t} \_\mathrm{R} * \mathrm{~S} \_$eqv to $X^{\prime}$ and $\mathrm{Y}$ is t_R*S_eqv to $Y^{\prime}$ then it may or may not be true that $X \cup Y$ is t_R*S_eqv to $X^{\prime} \cup Y^{\prime}$.

(ii) Sufficient condition for (i) to be true is that $X$ and $\mathrm{Y}$ are top $\mathrm{R}^{*} \mathrm{~S}$ comparable and $\mathrm{X}$ ' and $\mathrm{Y}^{\prime}$ are top $\mathrm{R} * \mathrm{~S}$ comparable.

(iii) The condition in (ii) is not necessary.

Proof follows as in the base case.

(6.1.7)(i) $\mathrm{X}$ is b_R*S_eqv to $\mathrm{X}^{\prime}$ and $\mathrm{Y}$ is $\mathrm{b} \_\mathrm{R} * \mathrm{~S}$ _eqv to $\mathrm{Y}^{\prime}$ may or may not imply that $X \cap Y$ is $\mathrm{b}_{-} \mathrm{R} * \mathrm{~S}_{-}$eqv to $X^{\prime} \cap Y^{\prime}$.

(ii) A sufficient condition for (i) to be true is that $\mathrm{X}$ and $\mathrm{Y}$ are bottom $\mathrm{R}^{*} \mathrm{~S}$ comparable and $\mathrm{X}^{\prime}$ and $\mathrm{Y}^{\prime}$ are bottom $R^{*} \mathrm{~S}$ comparable.

(iii) The condition in (ii) is not necessary.

Proof follows as in the base case. Here also we use Theorem 3.2.4. 
(6.1.8) (i) $\mathrm{X}$ is t_R*S_eqv to $\mathrm{Y}$ may or may not imply that $X \bigcup \sim Y$ is t_R* $\mathrm{S} \_$eqv to $\mathrm{U}$.

(ii) A sufficient condition for (i) to be true is that $\mathrm{X}$ is b_R*S_eqv to Y.

(iii) The condition in (ii) is not necessary.

Proof follows as in the base case.

(6.1.9) (i) $X$ is $b \_R^{*} S$ _eqv to $Y$ may or may not imply that $X \cap \sim Y$ is b_R*S_eqv to $\phi$.

(ii)A sufficient condition for (i) to be true is that $\mathrm{X}$ is t_R*S_eqv to Y.

(iii) The condition in (ii) is not necessary.

Proof follows as in the base case.

(6.1.10) If $X \subseteq Y$ and $\mathrm{Y}$ is $\mathrm{b} \_\mathrm{R} * \mathrm{~S} \_$eqv to $\phi$ then $\mathrm{X}$ is b_R*S_eqv to $\phi$.

(6.1.11) If $X \subseteq Y$ and $\mathrm{X}$ is $\mathrm{t} \_\mathrm{R} * \mathrm{~S} \_$eqv to $\mathrm{U}$ then $\mathrm{X}$ is t_R*S_eqv to U.

(6.1.12) $\mathrm{X}$ is $\mathrm{t} \_\mathrm{R} * \mathrm{~S} \_$eqv to $\mathrm{Y}$ iff $\sim X$ is $\mathrm{b} \_\mathrm{R} * \mathrm{~S} \_$eqv to $\sim Y$.

(6.1.13) $\mathrm{X}$ is b_R*S_eqv to $\phi$ and $\mathrm{Y}$ is $\mathrm{b} \_\mathrm{R} * \mathrm{~S} \_$eqv to $\phi \Rightarrow X \cap Y$ is $\mathrm{b} \_\mathrm{R} * \mathrm{~S} \_$eqv to $\phi$.

(6.1.14) If $X$ is $\mathrm{t} \_\mathrm{R} * \mathrm{~S}$ equv to $\mathrm{U}$ or $\mathrm{Y}$ is $\mathrm{t} \_\mathrm{R} * \mathrm{~S}$ _eqv to $\mathrm{U}$ then $X \cup Y$ is t_R*S_eqv to $\mathrm{U}$.

\subsection{Optimistic Multigranular Rough Equivalences}

Definition 6.2.1: Let $\mathrm{R}$ and $\mathrm{S}$ be two equivalence relations on $\mathrm{U}$ and $X, Y \subseteq U$. Then

(6.2.1) $\mathrm{X}$ and $\mathrm{Y}$ are optimistic bottom rough equivalent to each other with respect to $\mathrm{R}$ and $\mathrm{S}$ ( $\mathrm{X}$ Ob_R+S_eqv Y) if and only if $\underline{R+S} X$ and $\underline{R+S Y}$ are $\phi$ or not $\phi$ together.

(6.2.2) $\mathrm{X}$ and $\mathrm{Y}$ are optimistic top rough equivalent to each other with respect to $\mathrm{R}$ and $\mathrm{S}$ (X Ot_R+S_eqv $\mathrm{Y})$ if and only if $\overline{R+S} X$ and $\overline{R+S} Y$ are equal to $\mathrm{U}$ or not $\mathrm{U}$ together.

(6.2.3) $\mathrm{X}$ and $\mathrm{Y}$ are optimistic rough equivalent to each other with respect to $\mathrm{R}$ and $\mathrm{S}$ ( $\mathrm{X} \mathrm{O} \_\mathrm{R}+\mathrm{S} \_$eqv $\mathrm{Y}$ ) if and only if $\underline{R+S} X$ and $\underline{R+S Y}$ are $\phi$ or not $\phi$ together and $\overline{R+S} X$ and $\overline{R+S} Y$ are equal to $\mathrm{U}$ or not $\mathrm{U}$ together.

The following properties hold for optimistic Multigranular Rough Equivalences. In order to establish these properties we need the following additional definitions.
Definition 6.2.2: Let $K=(U, \mathfrak{R})$ be a knowledge base, $\mathrm{X}, \mathrm{Y} \subseteq \mathrm{U}$ and $\mathrm{R}, \mathrm{S} \in \mathrm{IND}(\mathrm{K})$. Then

(i) We say that $\mathrm{X}$ is bottom $(R+S)$-included in $\mathrm{Y}$ $\left(\mathrm{X} \subset_{R+S} \mathrm{Y}\right)$ if and only if $\underline{\mathrm{R}+\mathrm{SX}} \subseteq \underline{\mathrm{R}+\mathrm{SY}}$

(ii) We say that $\mathrm{X}$ is $\operatorname{top}(R+S)$-included in $\mathrm{Y}$ $\left(X \tilde{\subset}_{R+S} Y\right)$ if and only if $\overline{R+S} \mathrm{X} \subseteq \overline{R+S} \mathrm{Y}$.

(iii) We say that $\mathrm{X}$ is $(R+S)$-included in $\mathrm{Y}\left(X \tilde{\sim}_{\sim+S} Y\right)$ if and only if $\mathrm{X} \subset_{R+S} \mathrm{Y}$ and $X \tilde{\subset}_{R+S} Y$

Definition 6.2.3: (i) we say $\mathrm{X}, \mathrm{Y} \subseteq \mathrm{U}$ are bottom $R+S$ comparable if and only if $\mathrm{X}$ is bottom $(R+S)$-included in $\mathrm{Y}$ or $\mathrm{Y}$ is bottom $(R+S)$-included in $\mathrm{X}$.

(ii) We say $\mathrm{X}, \mathrm{Y} \subseteq \mathrm{U}$ are $\operatorname{top}(R+S)$ comparable if and only if $\mathrm{X}$ is top $(R+S)$-included in $\mathrm{Y}$ or $\mathrm{Y}$ is top $(R+S)$-included in $\mathrm{X}$.

(iii) We say $\mathrm{X}, \mathrm{Y} \subseteq \mathrm{U}$ are $(\mathrm{R}+\mathrm{S})$ comparable if and only if $\mathrm{X}$ and $\mathrm{Y}$ are both top and bottom $(\mathrm{R}+\mathrm{S})$ comparable.

\subsubsection{Properties}

(6.2.1.1) (i) If $X \cap Y$ is $\mathrm{b}_{-} \mathrm{R}+\mathrm{S} \_$eqv to $\mathrm{X}$ and $X \cap Y$ is b_R+S_eqv to $\mathrm{Y}$ then $\mathrm{X}$ is $\mathrm{b} \_\mathrm{R}+\mathrm{S} \_$eqv to $\mathrm{Y}$.

(ii) The converse of (i) is not necessarily true.

(iii) The converse cannot be true even if $\mathrm{X}$ and $\mathrm{Y}$ are bottom rough comparable.

(iv) The converse is true in (iii) if $\underline{\mathrm{RX}} \cup \underline{S} \mathrm{Y}=\mathrm{U}$ and $\underline{\mathrm{R} Y} \cup \underline{\mathrm{S} X}=\mathrm{U}$

(v) the conditions in (iv) are not neces sary.

Proof: (i) proof is trivial.

(ii) It is not true in the base case. So, using Theorem 3.2.6 it is not true in this case.

(iii) Even if $\mathrm{X}$ and $\mathrm{Y}$ are bottom rough comparable, we have $\underline{R+S}(X \cap Y) \subseteq \underline{R+S}(X)$ or $\underline{R+S Y}$ as the case may be. So, if both $\underline{R+S} X$ and $\underline{R+S Y}$ are $\phi$, we have $\underline{R+S}(X \cap Y)=\phi$. But when both are not $\phi$, we cannot say the same for $\underline{R+S}(X \cap Y)$.

(iv) Equality holds in (2.2.12) by theorem 3.2.6. So, the conclusion follows.

(v) The conditions in (iv) are not necessary as in the base case and the proof of theorem 3.2.4. 
(6.2.1.2) (i) If $X \cup Y$ is $\mathrm{t} \_\mathrm{R}+\mathrm{S} \_$eqv to $\mathrm{X}$ and $X \bigcup Y$ is $\mathrm{t} \_\mathrm{R}+\mathrm{S} \_$eqv to $\mathrm{Y}$ then $\mathrm{X}$ is $\mathrm{t} \_\mathrm{R}+\mathrm{S} \_$eqv to $\mathrm{Y}$.

(ii) The converse of (i) is not necessarily true.

(iii) The converse cannot be true even if $\mathrm{X}$ and $\mathrm{Y}$ are top rough comparable.

(iv) The converse is true in (iii) if $[\bar{R} X \cap \bar{S} Y]=\phi$ and $[\bar{R} Y \cap \bar{S} X]=\phi$.

(v) The conditions in (iv) are not necessary.

Proof: It is similar to the proof of (6.2.1.2). Only we note that under the conditions in (iv) equality holds in (2.2.13) by theorem 3.2.5 and hence the conclusion follows. The claim that the conditions in (iv) are not necessary is a consequence of the base case and the proof of theorem 3.2.5.

(6.2.1.3) (i) If $\mathrm{X}$ is $\mathrm{t} \_\mathrm{R}+\mathrm{S} \_$eqv to $X^{\prime}$ and $\mathrm{Y}$ is t_R+S_eqv to $Y^{\prime}$ then it may or may not be true that $X \cup Y$ is t_R+S_eqv to $X^{\prime} \cup Y^{\prime}$.

(ii) If $\mathrm{X}, \mathrm{Y}$ and $X^{\prime}, Y^{\prime}$ are top rough comparable and $\quad[\bar{R} X \cap \bar{S} Y]=\phi$ and $[\bar{R} Y \cap \bar{S} X]=\phi$ $\left[\bar{R} X^{\prime} \cap \bar{S} Y^{\prime}\right]=\phi$ and $\left[\bar{R} Y^{\prime} \cap \bar{S} X^{\prime}\right]=\phi$.

(iii) The conditions in (ii) are not necessary.

Proof: (i) follows from base case and Theorem 3.2.4. Even if $\mathrm{X}, \mathrm{Y}$ and $X^{\prime}, Y^{\prime}$ are rough comparable the conclusion may not be true. However, under the conditions of (ii) and Theorem 3.2.5 the proof follows.

(6.2.1.4) (i) If $\mathrm{X}$ is $\mathrm{b} \_\mathrm{R}+\mathrm{S} \_$eqv to $X^{\prime}$ and $\mathrm{Y}$ is b_R+S_eqv to $Y^{\prime}$ then it may or may not be true that $X \cap Y$ is t_R+S_eqv to $X^{\prime} \cap Y^{\prime}$.

(ii) If $\mathrm{X}, \mathrm{Y}$ and $X^{\prime}, Y^{\prime}$ are top rough comparable and $\underline{\mathrm{RX}} \cup \underline{\mathrm{S} Y}=\mathrm{U}$ and $\underline{\mathrm{R} Y} \cup \underline{\mathrm{S} X}=\mathrm{U}$

$\underline{\mathrm{R} X}{ }^{\prime} \cup \underline{S} Y^{\prime}=U$ and $\underline{R} Y^{\prime} U \underline{S} X^{\prime}=U$.

(iii) The conditions in (ii) are not necessary.

Proof: (i) follows from base case and Theorem 3.2.4. Even if $\mathrm{X}, \mathrm{Y}$ and $X^{\prime}, Y^{\prime}$ are rough comparable the conclusion may not be true. However, under the conditions of (ii) and Theorem 3.2.6 the proof follows.

(6.2.1.5) (i) $\mathrm{X}$ is $\mathrm{t} \_\mathrm{R}+\mathrm{S}$ _eqv to $\mathrm{Y}$ may or may not imply that $X \bigcup \sim Y$ is t_R+S_eqv to $\mathrm{U}$.

(ii) $\mathrm{X}$ is $\mathrm{b} \_\mathrm{R}+\mathrm{S} \_$eqv to $\mathrm{Y}$ is not a sufficient condition for (i) to be true. (iii) However, if $[\bar{R} X \cap \bar{S} Y]=\phi$ and $[\bar{R} Y \cap \bar{S} X]=\phi$ then (ii) is true.

(iv) The conditions in (iii) are not necessary.

Proof: (i) is not true by theorem 3.2.4 and base case.

(ii) The condition in (ii) is not sufficient as we have

$$
\begin{aligned}
\overline{R+S}(X \bigcup \sim Y) & \supseteq \overline{R+S}(X) \cup \overline{R+S}(\sim Y) \\
& =\overline{R+S}(X) \bigcup \overline{R+S}(\sim X) \\
& \subseteq \overline{R+S}(X \bigcup \sim X)=U .
\end{aligned}
$$

(iii) If the conditions in (iii) are further satisfied then equalities hold in place of inclusions in the above proof of (ii) and the conclusion is true.

(iv)The conditions are not necessary by the base case and theorem 3.2.4 and also, the theorem 3.2.5 .

(6.2.1.6) (i) $\mathrm{X}$ is b_R+S_eqv to $\mathrm{Y}$ may or may not imply that $X \cap \sim Y$ is t_R+S_eqv to $\phi$.

(ii) $\mathrm{X}$ is $\mathrm{t} \_\mathrm{R}+\mathrm{S} \_$eqv to $\mathrm{Y}$ is not a sufficient condition for (i) to be true.

(iii) However, if $\underline{\mathrm{RX}} \cup \underline{\mathrm{S} Y}=\mathrm{U}$ and $\underline{\mathrm{R} Y} \cup \underline{\mathrm{S} X}=\mathrm{U}$ then (ii) is true.

(iv) The conditions in (iii) are not necessary.

Proof: he proof is similar to (6.2.5).

The proofs of the following properties are similar to the base case.

(6.2.1.7) If $X \subseteq Y$ and $\mathrm{Y}$ is $\mathrm{b} \_\mathrm{R}+\mathrm{S} \_$eqv to $\phi$ then $\mathrm{X}$ is b_R+S_eqv to $\phi$.

(6.2.1.8) If $X \subseteq Y$ and $X$ is t_R+S_eqv to $\mathrm{U}$ then $\mathrm{Y}$ is $\mathrm{t} \_\mathrm{R}+\mathrm{S} \_$eqv to $\mathrm{U}$.

(6.2.1.9) $\mathrm{X}$ is $\mathrm{t} \_\mathrm{R}+\mathrm{S} \_$eqv to $\mathrm{Y}$ iff $\sim X$ is b_R+S_eqv to $\sim$.

(6.2.1.10) $\mathrm{X}$ is b_R+S_eqv to $\phi, Y$ is $b \_R+S \_$eqv to $\phi$ implies that $X \cap Y$ is b_R+S_eqv to $\phi$.

(6.2.1.11) If $X$ is $t \_R+S \_$eqv to $U$ or $Y$ is $t \_R+S \_e q v$ to $\mathrm{U}$ then $X \bigcup Y$ is $\mathrm{t} \_\mathrm{R}+\mathrm{S} \_$eqv to $\mathrm{U}$.

\subsection{Replacement properties for Multigranular approximate Equivalence}

We have stated above about the observations of Novotny and Pawlak (see for instance [5]) in connection with the holding of the properties for rough equality when the bottom and top equalities are 
interchanged. It is categorically told that the properties do not hold under this change. However, it was established by Tripathy et al $[9,10,11]$ that while some of these properties hold true after the interchanges are made, in some other cases additional sufficient conditions are necessary. These sufficient conditions may not be necessary for the results to be true. Similar properties were established for rough equivalence [18]. In this section we shall establish the replacement properties for multigranulations. First we take the case of pessimistic multigranulation in the next subsection

\subsubsection{Interchange Properties for Pessimistic Multigranular Equivalences}

(6.3.1.1) (i) If $X \cap Y$ is $\mathrm{t} \_\mathrm{R} * \mathrm{~S} \_$eqv to $\mathrm{X}$ and $X \cap Y$ is $\mathrm{t} \_\mathrm{R} * \mathrm{~S}$ _eqv to $\mathrm{Y}$ then $\mathrm{X}$ is $\mathrm{t} \_\mathrm{R} * \mathrm{~S} \_$eqv to $\mathrm{Y}$.

(ii) The converse of (i) is not necessarily true.

Proof: (i) trivial

(ii) Since $\overline{R * S}(X \cap Y) \subseteq \overline{R * S} X \cap \overline{R * S} Y$ the result fails to be true when $\overline{R * S} X=\overline{R * S Y}=U$ and $\overline{R * S}(X \cap Y) \neq U$.

(6.3.1.2)(i) If $X \cup Y$ is b_R*S_eqv to $\mathrm{X}$ and $X \cup Y$ is b_R*S_eqv to $Y$ then $X$ is $b \_R * S \_e q v$ to $Y$.

(ii) The converse of (i) is not neces sarily true.

Proof: (i) trivial.

(ii) Since $\underline{R * S}(X \cup Y) \supseteq \underline{R * S} X \cup \underline{R * S Y} Y$, the result fails to be true when $R * S X=R * S Y=\phi$ and $\underline{R * S}(X \cup Y) \neq \phi$.

(6.3.1.3) $\mathrm{X}$ is $\mathrm{b} \_\mathrm{R} * \mathrm{~S}$ eeqv to $X^{\prime}$, and $\mathrm{Y}$ is b_R*S_eqv to $Y^{\prime}$ may not necessarily imply that $X \cup Y$ is b_R*S_eqv to $X^{\prime} \cup Y^{\prime}$.

Proof: When $\underline{R * S} X, \underline{R * S} Y, \underline{R * S} X^{\prime}, \underline{R * S} Y^{\prime}$ are all $\phi$, one of $\underline{R * S}(X \cup Y)$ and $\underline{R * S}\left(X^{\prime} \cup Y^{\prime}\right)$ is $\phi$ but the other one is not $\phi$ the result fails to be true.

(6.3.1.4) $\mathrm{X}$ is $\mathrm{t} \_\mathrm{R} * \mathrm{~S} \_$eqv to $X$ ' and $\mathrm{Y}$ is $\mathrm{t} \_\mathrm{R} * \mathrm{~S} \_$eqv to $Y^{\prime}$ may not necessarily imply that $X \cap Y$ is t_R*S_eqv to $X^{\prime} \cap Y^{\prime}$.
Proof: Similar to (6.3.1.3).

(6.3.1.5) $\mathrm{X}$ is b_R*S_eqv to $\mathrm{Y}$ may or may not imply that $X \bigcup \sim Y$ is b_R*S_eqv to $\mathrm{U}$.

Proof: Follows from base case by theorem 3.2.4.

(6.3.1.6) $\mathrm{X}$ is $\mathrm{t} \_\mathrm{R} * \mathrm{~S}$ equ to $\mathrm{Y}$ may or may not imply that $X \cap \sim Y$ is t_R*S_eqv to $\phi$.

Proof: Follows from the base case and theorem3.2.4.

(6.3.1.7) If $X \subseteq Y$ and $\mathrm{Y}$ is $\mathrm{t} \_\mathrm{R} * \mathrm{~S} \_$eqv to $\phi$ then $\mathrm{X}$ is $\mathrm{t} \_\mathrm{R} * \mathrm{~S} \_$eqv to $\phi$.

Proof: By property as $X \subseteq Y$, we have $\overline{R * S} X \subseteq \overline{R * S} Y$. Also, here $\overline{R * S Y}=\phi$. So, the proof follows.

(6.3.1.8) If $X \subseteq Y$ and $\mathrm{X}$ is $\mathrm{b} \_\mathrm{R} * \mathrm{~S} \_$eqv $\mathrm{U}$ then $\mathrm{Y}$ is b_R*S_eqv U.

Proof: Here $\underline{R * S} X \subseteq \underline{R * S Y}$. Also by hypothes is $\underline{R * S} X=U$. So, the proof follows.

(6.3.1.9) $\mathrm{X}$ is $\mathrm{b} \_\mathrm{R} * \mathrm{~S} \_$eqv $\mathrm{Y}$ iff $\sim X$ is $\mathrm{t} \_\mathrm{R} * \mathrm{~S} \_$eqv to $\sim Y$.

Proof: We have $\underline{R * S} X$ and $\underline{R * S} Y$ are $\phi$ or not $\phi$ together. So, $\quad \overline{R * S}(\sim X)=\sim \underline{R * S X}$ and $\overline{R * S}(\sim Y)=\sim \underline{R * S Y}$ are $\mathrm{U}$ or not $\mathrm{U}$ together. Hence the proof follows.

(6.3.1.10) $\mathrm{X}$ is $\mathrm{t} \_\mathrm{R} * \mathrm{~S}$ _eqv to $\phi, \mathrm{Y}$ is $\mathrm{t} \_\mathrm{R} * \mathrm{~S}$ _eqv to $\phi \Rightarrow X \cap Y$ is t_R* $\mathrm{R}_{\text {_eqv }}$ to $\phi$.

Proof: This follows from the inclusion, $\overline{R * S}(X \cap Y) \subseteq \overline{R * S} X \cap \overline{R * S} Y$.

(6.3.2.11) $X$ is b_R*S_eqv to $U$ or $Y$ is $b \_R * S \_$eqv to $\mathrm{U} \Rightarrow X \bigcup Y$ is b_R* $\mathrm{R}_{-}$eqv to $\mathrm{U}$.

Proof: Here, $\underline{R * S} X=U$ or $\underline{R * S Y}=U$. So, the result follows from the inclusion

$$
\underline{R * S}(X \cup Y) \supseteq \underline{R * S} X \cup \underline{R * S} Y .
$$

\subsubsection{Interchange Properties for Optimistic Multigranular Equivalences}


(6.3.2.1) (i) If $X \cap Y$ is $\mathrm{t} \_\mathrm{R}+\mathrm{S} \_$eqv to $\mathrm{X}$ and $X \cap Y$ is $\mathrm{t} \_\mathrm{R}+\mathrm{S} \_$eqv to $\mathrm{Y}$ then $\mathrm{X}$ is $\mathrm{t} \_\mathrm{R}+\mathrm{S} \_$eqv to Y.

(ii) The converse of (i) is not necessarily true.

Proof: (i) trivial

(ii) Since $\overline{R+S}(X \cap Y) \subseteq \overline{R+S} X \cap \overline{R+S} Y$, the result fails to be true when $\overline{R+S} X=\overline{R+S} Y=U$ and $\overline{R+S}(X \cap Y) \neq U$.

(6.3.2.2)(i) If $X \bigcup Y$ is $\mathrm{b} \_\mathrm{R}+\mathrm{S} \_$eqv to $\mathrm{X}$ and $X \bigcup Y$ is b_R+S_eqv to $Y$ then $X$ is $b \_R+S \_$eqv to $Y$.

(ii) The converse of (i) is not necessarily true.

Proof: (i) trivial

(ii) Since $\underline{R+S}(X \cup Y) \supseteq \underline{R+S} X \cup \underline{R+S Y}$, the result fails to be true when $\underline{R+S} X=\underline{R+S Y}=\phi$ and $\underline{R+S}(X \cup Y) \neq \phi$.

(6.3.2.3) $\mathrm{X}$ is b_R+S_eqv to $X$ ' and $\mathrm{Y}$ is $\mathrm{b} \_\mathrm{R}+\mathrm{S} \_$eqv to $Y^{\prime}$ may not necessarily imply that $X \cup Y$ is b_R+S_eqv to $X^{\prime} \cup Y^{\prime}$.

Proof: When $\underline{R+S} X, \underline{R+S Y}, \underline{R+S} X^{\prime}, \underline{R+S Y^{\prime}}$ are all $\phi$, one of $\underline{R+S}(X \cup Y)$ and $\underline{R+S}\left(X^{\prime} \cup Y^{\prime}\right)$ is $\phi$ but the otherone is not $\phi$ the result fails to be true.

(6.3.2.4) $X$ is $t \_R+S \_$eqv to $X$ ' and $Y$ is $t \_R+S \_$eqv to $Y^{\prime}$ may not necessarily imply that $X \cap Y$ is t_R+S_eqv to $X^{\prime} \cap Y^{\prime}$.

Proof: Similar to (6.3.2.3).

(6.3.2.5)X is b_R+S_eqv to $\mathrm{Y}$ may or may not imply that $X \bigcup \sim Y$ is b_R+S_eqv to $\mathrm{U}$.

Proof: Follows from the base case and theorem 3.2.4.

(6.3.2.6) $\mathrm{X}$ is $\mathrm{t} \_\mathrm{R}+\mathrm{S} \_$eqv to $\mathrm{Y}$ may or may not imply that $X \cap \sim Y$ is t_R+S_eqv to $\phi$.

Proof: Follows from the base case and theorem 3.2.4.

The proofs of the following properties are similar to those in (6.3.1.7) to (6.3.1.11).
(6.3.2.7)If $X \subseteq Y$ and $\mathrm{Y}$ is $\mathrm{t} \_\mathrm{R}+\mathrm{S} \_$eqv to $\phi$ then $\mathrm{X}$ is t_R+S_eqv to $\phi$.

(6.3.2.8)If $X \subseteq Y$ and $\mathrm{X}$ is $\mathrm{b} \_\mathrm{R}+\mathrm{S} \_$eqv $\mathrm{U}$ then $\mathrm{Y}$ is b_R+S_eqv U.

(6.3.2.9) $\mathrm{X}$ is $\mathrm{b} \_\mathrm{R}+\mathrm{S} \_$eqv $\mathrm{Y}$ iff $\sim X$ is $\mathrm{t} \_\mathrm{R}+\mathrm{S} \_$eqv to $\sim Y$.

(6.3.2.10) $\mathrm{X}$ is $\mathrm{t} \_\mathrm{R}+\mathrm{S}$ _eqv to $\phi, \mathrm{Y}$ is $\mathrm{t} \_\mathrm{R}+\mathrm{S}$ eqv to $\phi \Rightarrow X \cap Y$ is t_R+S_eqv to $\phi$.

(6.3.2.11) $X$ is b_R+S_eqv to $U$ or $Y$ is $b \_R+S \_$eqv to $\mathrm{U} \Rightarrow X \cup Y$ is b_R+S_eqv to $\mathrm{U}$.

\section{Conclusion}

In this paper we introduced the concepts of optimistic multigranular equivalences and pessimistic multigranular equivalences. These concepts extend the approximate equivalences for single granulation and lead to approximate reasoning to the level of multigranulations. We have provided an example to show how the approximate equivalences use human knowledge for reasoning and how these are better than the corresponding approximate equalities considered in [20]. Several properties which are true for the single granulation case have been extended suitably and proved. Also, the replacement properties have been extended to this general setting.

\section{References}

[1] Novotny, M. and Pawlak, Z.: Characterization of Rough Top equalities and Rough Bottom Equalities", Bull. Polish Acad. Sci. Math., 33, 1985, 91-97.

[2] Novotny, M. and Pawlak, Z.: On Rough Equalities, Bull. Polish Acad. Sci. Math., 33, 1985, 99-104.

[3] Novotny, M. and Pawlak, Z.: Black Box Analysis and Rough Top Equality", Bull. Polish Acad. Sci. Math., 33, 1985, 105-113.

[4] Pawlak, Z.: Rough Sets, International Journal of Information and Computer Science, 1982, 341-346.

[5] Pawlak, Z.: Rough Sets, Theoretical Aspects of Reasoning about Data, Kluwer Academic Publishers, 1991.

[6] Pawlak, Z. and Skowron, A.: Rudiments of rough sets, Information Sciences-An International Journal, Elsevier Publications, 177(1), 2007, 3-27.

[7] Pawlak, Z. and Skowron, A.: Rough sets: Some Extensions, Information Sciences, V01.177, 2007, $28-40$. 
[8] Qian, Y.H and Liang, J.Y.: Rough set method based on Multi-granulations, Proceedings of the 5th IEEE Conference on Cognitive Informatics, vol.1, 2006, $297-304$.

[9] Qian, Y.H., Liang, J.Y and Dang, C.Y.: Pessimistic rough decision, in: Proceedings of RST 2010, Zhoushan, China, 2010, 440-449.

[10] Tripathy, B.K.: Rough sets on Intuitionistic fuzzy approximation spaces, Notes on Intuitionistic Fuzzy Sets, (Bulgaria), vol.12, no.1, 2006, 45-54.

[11] Tripathy, B.K.: On Approximation of classifications, rough equalities and rough equivalences, Studies in Computational Intelligence, vol.174, Rough Set Theory: A True Land mark in Data Analysis, Springer Verlag, 2009, $85-136$.

[12] Tripathy, B.K.: Rough sets on Fuzzy approximation spaces and Intuitionistic Fuzzy approximation spaces", Springer International studies in computational intelligence, vol.174, Rough Set Theory: A True landmark in Data Analysis, Ed: A. Abraham, R.Falcon and R.Bello, (2009), pp.3 - 44.

[13] Tripathy, B.K.: An Analysis of Approximate Equalities based on Rough Set Theory, International Journal of Advanced Science and Technology Vol. 31, June, 2011, pp.23 -36.

[14] Tripathy, B.K., Mitra, A. and Ojha, J.: On Rough Equalities and Rough Equivalences of Sets, SCTC 2008-Akron, U.S.A., Springer-Verlag Berlin Heidelberg (2008), LNAI 5306, pp. 92-102.

[15] Tripathy, B.K., Mitra, A. and Ojha, J.: Rough Equivalence and Algebraic properties of Rough Sets, International Journal of Artificial Intelligence and Soft Computing, (Switzerland), vol.1, nos.2/3/4, 2009, $271-289$.

[16] Tripathy, B.K. and Gantayat, S.S.: Rough Sets on Fuzzy Similarity relations and Applications to Information retrieval, International Journal of Fuzzy Systems and Rough Systems (IJFSRS), January-June 2010, Volume 3, Is sue 1, 1-13.

[17] Tripathy, B.K. and Nagaraju, M.: A comparative analysis of multigranular Approaches and on topological properties of Incomplete Pessimistic Multigranular Rough Fuzzy Sets, International Journal of Intelligent Systems and Applications, vol.11, 2012, 99-109.

[18] Tripathy, B.K. and Raghavan, R.: On Some Comparison Properties of Rough Sets Based on Multigranulations and Types of Multigranular Approximations of Classifications, Accepted for publication in the International journal of intelligent systems and Applications, 2013.

[19] Tripathy, B.K. and Raghavan, R.: Some Algebraic properties of Multigranulations and an Analys is of
Multigranular Approximations of Classifications, Accepted for publication in the International Journal of Information Technology and Computer Science, 2013.

[20] Tripathy, B.K. and Mitra, A.: On the Approximate Equalities of Multigranular Rough Sets and Approximate Reasoning, (accepted for) Springer ACITY 2013:international conference, Chennai, India, 2013.

\section{Authors' Profiles}

B. K. Tripathy: Senior Professor in the school of computing sciences and engineering, VIT University, at Vellore, India, has published more than 170 technical papers in international journals/ proceedings of international conferences/ edited book chapters of reputed publications like Springer, IGI and has guided 12 students for $\mathrm{PhD}$, so far. $\mathrm{He}$ is having more than 30 years of teaching experience. $\mathrm{He}$ is a member of international professional associations like IEEE, ACEEE, CSTA, ACM, IRSS, CSI, IMS, OITS, OMS, IACSIT, IST and is a reviewer of around 35 international journals which include World Scientific, Springer, Science Direct publications and IEEE. Also, he is in the editorial board of at least 12 international journals. His current research interest includes Fuzzy sets and systems, Rough sets and knowledge engineering, Granular computing, soft computing, bag theory, list theory, Content based learning, Remote laboratories and social network analysis.

Anirban Mitra: Asst. Professor in the Department of Computer Science Engineering, M.I.T.S., Rayagada, Odisha, India, has co-authored and published several papers in journals and conferences. He is pursuing Ph.D. in Computer Science, from Berhampur University, Berhampur, Odisha, India under the guidance of Dr. B. K. Tripathy. He did his Master's in Computer Applications. His research areas are Rough Set, Granular Computing and Social Network.

How to cite this paper: B. K. Tripathy, Anirban Mitra,"On Approximate Equivalences of Multigranular Rough Sets and Approximate Reasoning", International Journal of Information Technology and Computer Science(IJITCS), vol.5, no.10, pp.103-113, 2013. DOI: 10.5815/ijitcs.2013.10.11 